\title{
Alter
}

Revue de phénoménologie

$21 \mid 2013$

La Vie

\section{Le « mouvement qui trace et laisse derrière lui les figures du sillage »}

\section{Lucia Angelino}

\section{(2) OpenEdition}

\section{Journals}

Édition électronique

URL : http://journals.openedition.org/alter/898

DOI : $10.4000 /$ alter.898

ISSN : 2558-7927

Éditeur :

Association ALTER, Archives Husserl (CNRS-UMR 8547)

\section{Édition imprimée}

Date de publication : 1 novembre 2013

Pagination : 279-297

ISBN : 978-2-95-223749-9

ISSN : $1249-8947$

\section{Référence électronique}

Lucia Angelino, « Le « mouvement qui trace et laisse derrière lui les figures du sillage » », Alter [En ligne], 21 | 2013, mis en ligne le 01 juin 2019, consulté le 05 juillet 2019. URL : http:// journals.openedition.org/alter/898; DOI : 10.4000/alter.898 


\title{
LE « MOUVEMENT QUI TRACE ET LAISSE DERRIÈRE LUI LES FIGURES DU SILLAGE $»^{1}$
}

\author{
Lucia Angelino
}

\begin{abstract}
On demande : où l'histoire se fait-elle ? [...] Quel est ce mouvement qui trace et laisse derrière lui les figures du sillage? Il est du même ordre que le mouvement de la Parole et de la Pensée, et enfin que l'éclatement $d u$ monde sensible entre nous: partout il y a sens, dimensions, figures par-delà ce que "chaque conscience" aurait pu produire $[\ldots]^{2}$.
\end{abstract}

Cette interrogation et cette ébauche de réponse nous placent au cour de la conception merleau-pontienne du mouvement de l'expression, le mouvement comme force lisible à même les formes (le sensible) et comme dynamisme figural intrinsèque à l'espace métaphorique de la pensée. Cette conception du mouvement a, chez Merleau-Ponty, une portée considérable. Elle occupe une position à ce point cardinale que l'on peut, à travers son examen, déceler la formule charnelle la plus secrète du mouvement (dont le changement de lieu n'est qu'une définition superficielle et particulière, restreinte): le mouvement comme vibration d'une énergie qui germe dans l'apparence (la chair du visible) et nous communique l'imaginaire pulsation de la vie. Telle est, du moins, la thèse dont je voudrais me faire ici l'interprète. Elle tend à montrer que, à travers les réflexions éparses que Merleau-Ponty consacre au mouvement pictural, on voit émerger une définition plus

\footnotetext{
${ }^{1}$ M. Merleau-Ponty, Signes (1960), Paris, Gallimard, 2003, p. 36. Dorénavant noté S. On serait tenté de suggérer qu'il fallut toute l'imagination et l'habilité technique d'un Vélasquez « pour découvrir le moyen de suggérer le mouvement par cet "éclat incertain" dans le cercle du rouet du tableau Les Fileuses qui paraît saisir ce que l'on nomme l'"effet stroboscopique", le sillage indistinct que laisse dans le champ de vision le passage d'un objet en mouvement ", E. H. Gombrich, L'Art et l'illusion, Paris, Gallimard, 1996, p. 191.

$2 S$, p. 36.
} 
riche et fine du mouvement, qui réunit les deux significations de la dunamis aristotélicienne: potentia passiva, poussée génératrice, ou mouvement-expression-vibration-rayonnement et potentia activa, tension vers l'acte, ou mouvement-geste. Peu à peu, on comprendra que cette double intuition du mouvement est nécessairement liée à une conception radicalement dynamique de l'être - autre nom pour dire l'apparaître - et de l'attitude que l'homme doit adopter pour le sentir et le connaitre ${ }^{3}$. De proche en proche, on verra que ce mode d'appréhension du mouvement s'avère d'un enjeu stratégique pour penser à la fois le devenir expressif de la chair du monde et l'avènement de l'histoire qui naît en son sein, l'acte (geste) qui métamorphose la nature en cuvre humaine.

1. Penser les mouvements pour eux-mêmes: retour du mouvement humain au mouvement des êtres naturels. Le mouvement comme rayonnement d'une énergie qui germe dans l'apparence et nous communique l'imaginaire pulsation de la vie.

Dans cette première partie, je propose de faire émerger la nouvelle intuition du mouvement à l'œuvre dans la pensée de Merleau-Ponty, en prêtant attention aux passages consacrés au mouvement - en principe immobile -, qui est inhérent à l'espace pictural, aux formes perçues en peinture (artistiques). Il est étonnant de constater en effet que le problème du mouvement trouvera peu à peu son point de condensation théorique, sa signification la plus secrète et la plus profonde à partir de l'examen des mouvements des êtres naturels (représentés en peinture) - tel le mouvement des vagues et des nuages, l'ondoiement des arbres et le déploiement des feuillages, les vibrations d'un tissu, comme le battement d'une draperie, ou l'ondulation d'une nappe blanche... ${ }^{4}$

\footnotetext{
${ }^{3}$ Cf. le passage suivant de la Préface à Signes : «On rappelait récemment le mot de Montaigne "tout mouvement nous découvre" et l'on en tirait avec raison que l'homme n'est qu'en mouvement. De même le monde ne tient, l'Être ne tient qu'en mouvement, c'est ainsi seulement que toutes choses peuvent être ensemble », S, p. 39.

${ }^{4}$ Cf. à titre d'exemple le passage suivant: "Balzac décrit dans la Peau de Chagrin une "nappe blanche comme une couche de neige fraîchement tombée et sur laquelle s'élevaient symétriquement les couverts couronnés de petits pains blonds". "Toute ma jeunesse, disait Cézanne, j'ai voulu peindre ça, cette nappe de neige fraîche... Je sais maintenant qu'il ne faut vouloir peindre que : s'élevaient symétriquement les couverts, et: les petits pains blonds. Si je peins : couronnés, je suis foutu, comprenez-vous? Et si vraiment j'équilibre et je nuance mes couverts et mes pains comme sur nature, soyez sûr que les couronnes, la neige et tout le tremblement y seront" ", Merleau-Ponty, Phénoménologie de la perception (1945), Paris, Gallimard, 2003, p. 230 et également les passages consacrés au mouvement de l'expression, à ce moment
} 
Le mouvement ayant lieu dans l'immobile, autrement dit le mouvement figuré à travers la dynamique des formes, des éléments déplacés sur la surface de la toile, s'avère en effet d'un enjeu stratégique pour celui qui pointe la formule charnelle et la plus secrète du mouvement, puisqu'il nous introduit au cœur même d'une mobilité plus essentielle qui est la mobilité même de l'apparaître, « la vibration des apparences, qui est le berceau des choses $»^{5}$, selon les termes propres de Paul Cézanne : expression d'une présence intensive, révélation d'un sens immanent ou naissant à même les corps - tant animés qu'inanimés - rayonnement, vibration d'une énergie interne à son effectuation qui germe dans l'apparence et nous communique l'imaginaire pulsation de la vie.

Le battement d'une draperie sous la poussée du vent, l'ondulation d'une chevelure dans l'air, les vibrations des feuillages... tout cela lui aura permis en outre de penser l'abandon du corps à un mouvement extérieur - le mouvement de l'air, du vent, du feu, de l'eau... - qui le précède et dans lequel il doit s'insérer pour s'ouvrir à la passion d'un être mû : quelque chose comme la poussée d'un mouvement intérieur, doublée de l'exposition à des mouvements extérieurs (une mobilité intérieure ressentie au contact des mouvements extérieurs), autrement dit la double dimension vécue et agie d'un mouvement qui s'accomplit au point de contact entre notre corps et la chair du monde et qui, en ce sens, exprime la correspondance vécue entre les rythmes de la nature (les rythmes biologiques) et les rythmes qui habitent et meuvent notre propre corps, la conjonction rythmique d'un se mouvoir et d'un être $m \hat{u}^{6}$.

En un mot, le mouvement dans l'art reconduit le philosophe à la signification la plus profonde et secrète du mouvement, dont le changement de lieu n'est qu'une formule finale et superficielle: le mouvement comme force et comme énergie rayonnante depuis l'image,

\footnotetext{
énigmatique où les choses réalisent le miracle de l'expression : «Avant autrui, écrit-il, la chose réalise ce miracle de l'expression : un intérieur qui se révèle au dehors, une signification qui descend dans le monde et se met à y exister et qu'on ne peut comprendre pleinement qu'en la cherchant du regard en son lieu », Ibid., p. 369.

${ }^{5}$ M. Merleau-Ponty, « Le doute de Cézanne », in Sens et non-sens (1948), Paris, Gallimard, 1996.

${ }^{6} \mathrm{~L}$ 'on comprend mieux par là que cette théorie du mouvement trouve à s'exprimer davantage et bien plus profondément dans les mouvements des êtres naturels - tel le battement d'une draperie, l'ondulation d'une chevelure, le ploiement d'une branche - que dans les êtres corporels, précisément parce que pour eux, être en mouvement, c'est être traversé, voire affecté par un élément différentiel et/ou hétérogène: le pathos de la vie elle-même. Textiles, cheveux, feuilles, drapés, branchages, toutes ces choses inanimées se trouvent emportées, elles ne possèdent pas une force motrice propre, mais doivent s'ouvrir à la passion d'un être mî. Et pourtant leur abandon à ce mouvement extérieur nous fait signe, ou mieux encore nous fait un geste.
} 
comme manifestation (ou révélation) d'une force interne à son effectuation qui l'habite et en est inséparable, le mouvement comme mode d'être sous lequel apparaît tout être vivant, pouvant prendre la double forme d'une disponibilité passive à subir un mouvement extérieur ou $\mathrm{d}$ 'une capacité active à réaliser un nouveau mouvement.

Il revient sans doute aux grands historiens de l'art - à commencer par Bernard Berenson, auquel se réfère Merleau-Ponty - de l'avoir exprimé. Ainsi, comme ces quelques lignes le posent clairement,

Le mouvement qui, après les valeurs tactiles, est l'élément le plus essentiel de l'œuvre d'art, n'a rien à voir avec le changement de place, ou même avec le changement d'attitude ou de pose, et encore moins avec l'activité transitive sous toutes ses formes. Le mouvement, c'est la nette énergie qui donne vie aux arabesques qui limitent une cuvre et au dessin de toutes les parties qui se trouvent entre ces limites. Une arabesque ou un dessin qui devient énergique c'est ce qu'on appelle contour. [...] Cela ressemble à ce que nous voyons dans les cours d'eaux rapides mais paisibles, où les remous et les tourbillons produits par le courant ne varient pas tant que durent les mêmes conditions; et cependant il n'est pas une goutte d'eau qui contribue à façonner ces dessins aquatiques, dont on ne puisse dire qu'elle soit la même pendant deux secondes consécutives'.

En un mot fulgurant, tel le résume Merleau-Ponty,

Le mouvement en peinture n'a rien à voir avec le changement de place ou même avec le changement d'attitude ou de pose, et encore moins avec l'activité transitive sous toutes les formes. [...] Le mouvement c'est l'énergie qui habite (naturellement) une ligne comme les mouvements de l'eau habitent un tourbillon quoiqu'il dessine une figure constante... ${ }^{8}$

Pour séduisante qu'elle soit, la définition du mouvement évoquée jusqu'ici demande à être questionnée, si bien qu'elle pose un véritable paradoxe, celui d'un mouvement sans déplacement, figé dans l'imminence d'un instant, d'un instantané, pourtant révélateur de l'énergie vitale qui l'habite et qui caractérise déjà chez Aristote (Physique) l'être $\mathrm{du}$ mouvement dans la nature ou dans la physis ${ }^{9}$, l'état intérieur

\footnotetext{
${ }^{7}$ Bernard Berenson, Esthétique et Histoire des arts visuels, Paris, Éditions Albin Michel, 1953, p. 87.

${ }^{8}$ Cité par Merleau-Ponty, Le Monde sensible et le monde de l'expression, Genève, Metis Presses, 2011, p. 166, dorénavant noté MSME.

${ }^{9}$ Nous retrouvons ici le noyau essentiel de la conception du mouvement selon Aristote. Comme le souligne de manière éclairante Heidegger dans un bref passage du texte intitulé Vom Wesen und Begriff der physis, Aristoteles, Physik B, 1, (1939), en français De l'essence et du concept de physis, Aristote, Physique B, 1: nous ne pouvons saisir le noyau essentiel du mouvement pensé de manière grecque, que si nous observons que dans le changement apparaît quelque chose qui était jusque-là voilé, invisible ou absent («jaillissement, éclosion » [Aus-schlag] et «éclat, irruption,
} 

d'énergie que nous saisissons dans chaque corps se trouvant en mou-
vement.

Autrement dit, ce n'est pas, à proprement parler, le mouvement lui-même que la peinture peut représenter, mais ce qui, dans le mouvement, ne se meut pas ou "s'y maintient fixe ${ }^{10}(S, \mathrm{p}$. 39), cette partie immobile du mobile que constitue le lieu, ce lieu ayant pourtant la signification d'un instant, d'un instantané du corps, d'un arrêt idéal où il prend sa forme, se singularise, se compose en une figure corrélative de sa circonscription sur le fond continu de la toile. Ce n'est donc pas le mouvement lui-même que la peinture peut représenter, mais plutôt la puissance qui fait le mouvement, la dunamis toujours coprésente à l'energeia sous le mode d'un mouvement non encore réalisé11.

Un tel paradoxe - celui d'un mouvement sans déplacement, ayant le sens d'une force, d'une énergie rayonnante depuis l'image - est pourtant bien réel, en tant qu'il correspond à notre expérience charnelle (perceptive) du mouvement perçu, celle d'une énergie qui «s'accompagne

apparition, attestation fulgurante " [Durchshlag]). Aujourd'hui, nous devons réussir à faire deux choses : 1 . Nous libérer de l'idée courante selon laquelle le mouvement est en premier lieu un déplacement, voire un changement de lieu ; 2 . Apprendre à voir que le mouvement, en tant que modalité, voire manifestation de l'être, a, selon les Grecs, une tout autre signification, le caractère d'un se montrer ou d'un s'exprimer, d'un venir au paraître. Cf. M. Heidegger, "Vom Wesen und Begriff der physis, Aristoteles, Physik B, 1 (1939) », in Gesamtausgabe I. Abteilung : Veröffentlichte Schriften 1914-1970, Band 9 Wegmarken, Francfort-sur-le-Main, Klostermann, 1976, p. 239-303. La traduction française de ce texte n'existe pas encore. Je propose ici une traduction du passage cité qui se trouve à la page 249 de l'édition allemande.

Cf. également à ce sujet R. Bernet, "La négativité et la contrariété des pulsions (Heidegger et Aristote) ", Alter, no 14, 2006, p. 65-86. Voir en particulier le paragraphe I, intitulé "La structure du mouvement et la particularité des mouvements naturels", p. 67-74.

${ }^{10} \mathrm{Cf}$. le passage suivant de la Préface à Signes : «On dit invisible comme on dit immobile : non pour ce qui est étranger au mouvement, mais pour ce qui s'y maintient fixe. C'est le point ou le degré zéro de visibilité, l'ouverture d'une dimension de visibilité », S, p. 39.

${ }^{11}$ Comme le remarque de manière percutante Jan Patočka, avec le couple de concepts aristotéliciens de dunamis-energeia, "désignant d'abord l'aptitude ou la force et sa manifestation, puis, dans une acception plus large, la possibilité et l'acte effectif ", l'on en arrive ainsi, au terme d'un long cheminement, «à la possibilité de définir effectivement le mouvement, de cerner son essence unitaire, sa détermination ontologique», de saisir ce qu'est le mouvement dans son fond, à savoir une possibilité qui se réalise, ou la réalisation de la dunamis, «La conception aristotélicienne du mouvement : signification philosophique et recherches historiques ", in Le Monde naturel et le mouvement de l'existence humaine, Dordrecht, Kluwer Academic Publishers, 1988, p. 137. Toutefois, puisque «Aristote localise la puissance dans un substrat censé rendre le changement possible en subsistant sans changement", seule "pourrait faire avancer la question une radicalisation de la conception aristotélicienne - le mouvement comme vie originelle qui ne reçoit pas son unité du substrat conservé, mais crée elle-même sa propre unité et celle de la chose en mouvement. Seul le mouvement conçu de cette manière est mouvement originel », Jan Patočka "Méditation sur "Le Monde naturel comme problème philosophique" ", in Le Monde naturel et le mouvement de l'existence humaine, op. cit., p. 103. 
d'un mouvement des yeux et sollicite notre capacité d'Einfühlung, tend à un sentiment d'identification esthétique $»^{12}$, en tant qu'elle " fait vibrer notre regard, virtuellement notre toucher et nos oreilles ${ }^{13}$.

Il suffit, pour s'en apercevoir, de se situer du point de vue de l'image. Elle nous transmet une force et une énergie qui résulte du dynamisme des éléments qu'y co-paraissent ( $\mathrm{du}$ mouvement figural). Elle est chargée d'une énergie et d'une tension dynamique qui fait littéralement lever le potentiel de la sensibilité et de la mémoire du spectateur qui la reçoit. Elle n'est pas quelque chose d'immobile et d'autonome, elle n'est pas un archétype. Elle ne se sépare pas $d u$ dynamisme du geste qui l'a produite. Elle jaillit comme un éclair dans la mémoire involontaire et renvoie toujours au-delà d'elle-même à un tout dont elle fait partie.

$C^{\prime}$ est une charge émotive et dynamique de ce genre qu'on voit bien à l'œuvre dans les premiers traits peints à Lascaux, qui sont à l'origine même de l'histoire de l'art ${ }^{14}$. Ces peintures, ces figures, devant nous, sont miraculeuses, elles nous communiquent une émotion forte et intime. Mais elles sont d'autant plus inintelligibles que rien ne nous renseigne davantage sur la place précise que ces figures ont eue dans les croyances et dans les mythes des hommes qui les ont peintes, et qui vécurent bien des millénaires avant l'Histoire. Et pourtant ces figures nous émeuvent profondément. C'est la vie, l'éclat, le mouvement et son caractère suspendu (retenu, contradictoire), la gestualité - elle seule - de ces figures qui nous étonne, qui nous donne même le sentiment de la vie. Corps réels, creusés d'un vide, d'une trace marquée dans la pierre, les animaux de Lascaux encourent le risque d'un déséquilibre, d'un suspens, qui soulève leurs corps du sol des parois et qui nous sollicite corporellement à ressentir le pathos d'une expérience qui, pour les chasseurs, était fondamentale : l'expérience d'une verticalité qui les séparait désormais du passé de leur vie animale. Ce qui nous touche à Lascaux, c'est la beauté qui émane des mouvements fiévreux et libres des animaux qui se poursuivent fabuleusement de paroi en paroi, c'est le langage expressif des gestes qui produit l'intensité, à la fois physique et affective. Nous voyons à Lascaux une cavalcade animale se poursuivant sur les parois. Et c'est précisément le mouvement qui anime les grands taureaux dans la fresque, qui nous

\footnotetext{
12 B. Berenson, Esthétique et Histoire des arts visuels, op. cit, p. 87.

${ }^{13}$ La Prose du monde (1969), Paris, Gallimard, 2004, p. 192, dorénavant noté PM.

${ }^{14} \mathrm{Cf}$. à ce sujet le magnifique texte de Georges Bataille intitulé Lascaux ou la naissance de l'art, in CEuvres complètes IX, Paris, Gallimard, 1979, p. 9-101.
} 
donne un sentiment fort de leur présence, un sentiment de force et de grandeur lisible à même le mouvement et son suspens.

$C^{\prime}$ est ce même sentiment de présence, de claire et brûlante présence que nous donnent les chefs-d'œuvre de tous les temps. C'est là que réside l'essentiel. L'essentiel est de comprendre que la force du trait tient au geste qui le porte et qui le trace. Depuis les plus anciens vestiges des époques aurignaciennes ou magdaléniennes gravés sur les rochers, jusqu'aux œuvres les plus modernes, c'est bien le geste, la mobilité qui nous touche et rend présente l'œuvre, quelle que soit son époque.

2. De l'immobilité de la statue au mouvement ayant lieu dans l'immobile et de celui-ci à la détermination ontologique du mouvement

Dès lors, la question se pose de savoir comment la peinture, les arts visuels en général peuvent faire voir, jusque dans l'immobilité de l'image, l'energeia, la force, la puissance en laquelle consiste le mode d'être (et d'apparaître) fondamental de l'être-en-mouvement : comment la peinture - «poésie muette », muta poesia, selon le mot de Léonard - peutelle nous rendre présente, l'éloquence des gestes, nous donner à voir, jusque dans l'immobilité de l'image, le mouvement?

Dans un bref passage de L'cil et l'esprit, Merleau-Ponty nous livre l'essentiel de son intuition :

Ce qui donne le mouvement, dit Rodin, c'est une image où le bras, les jambes, le tronc, la tête sont pris chacun à un autre instant, qui donc figure le corps dans une attitude qu'il n'a eue à aucun moment, et impose entre ces parties des raccords fictifs, comme si cet affrontement d'incompossibles pouvait seul faire sourdre dans le bronze et sur la toile la transition et la durée ${ }^{15}$.

En d'autres termes, ce qui donne le mouvement en peinture, c'est la co-présence paradoxale de la dunamis déjà réalisée - d'où il résulte - et de la dunamis encore dormante - qu'il porte en lui sous la forme d'une potentialité non encore actualisée, d'un mouvement en train de s'accomplir, qui frémit déjà en quelque sorte dans celui qui le précède. Peut-être n'y a-t-il d'autre définition du mouvement que celle-là : quelque chose comme l'insertion d'un mouvement dans un autre "mouvement qui le précède» et "qui a déjà commencé dans son

\footnotetext{
${ }^{15}$ M. Merleau-Ponty, L'ceil et l'esprit (1964), Paris, Gallimard Folioplus , 2006, p. 53-54, dorénavant noté $O E$.
} 
dos $»^{16}$. En sorte que tout mouvement résulterait de la jointure mouvante de deux mouvements au moins. En réalité, il faudrait aller plus loin encore et penser tout mouvement comme un montage dont les éléments ne sont pas juxtaposés ou additionnés, mais organiquement joints et mis en mouvement par un travail qui «se prémédite entre les jambes, le tronc, les bras, la tête, en quelque foyer virtuel, et il n'éclate qu'ensuite en changement de lieu » $(O E$, p. 54).

Tout mouvement suppose en effet toujours un rapport, une rencontre, un mélange paradoxal entre une dunamis pressante (agissante, présente) et une energeia non encore pleinement réalisée et même la commotion "de motions et d'émotions affrontées d'un seul geste agi ${ }^{17}$. Voilà pourquoi Merleau-Ponty en parle comme d'un "affrontement d'incompossibles ", pour souligner précisément le caractère différentiel de tout mouvement ne pouvant s'effectuer que s'il danse un pas de deux avec un autre mouvement idéel et non actuel à celui-là.

Encore faut-il remarquer que cette co-présence paradoxale, cet affrontement d'incompossibles suppose pourtant toujours une absence, un intervalle, un écart différentiel entre les deux, une puissance d'arrêt qui est le véritable moteur dans le passage, ou dans le changement (basculement) de la dunamis à l'energeia. Ce n'est pas seulement une pause, c'est une disjonction, un suspens tendu entre l'avant et l'après, quelque chose comme une hésitation prolongée que seul le ralenti de la caméra pourra nous restituer. Il ne s'agit donc pas d'une pause au sens chronologique, c'est plutôt une puissance d'arrêt qui travaille l'image ellemême, qui la soustrait au pouvoir narratif (au flux continu) pour l'exposer en tant que telle. Il y a là de profondes analogies avec l'arrêt (découpage) constitutif de la technique du montage dans le cinéma d'une part et d'autre part avec la césure en poésie qui, arrêtant le rythme et le déroulement des images, fait apparaître le mot et la représentation en tant que tels. Arrêter le mot, de même que représenter «ce mouvement que l'on dit accompli lorsqu'il y a eu changement de lieu ${ }^{18}$, c'est le soustraire au flux du sens, pour l'exhiber en tant que tel.

Il y a donc - pourrait-on dire - deux conditions du mouvement en peinture : les césures et les enjambements, l'arrêt et la composition. Ces deux modalités de l'être-en-mouvement se conjuguent déjà au sein de la

\footnotetext{
16 Selon une heureuse expression que nous empruntons à B. Prévost, La peinture en actes, Arles, Actes Sud, 2007, p. 73.

${ }^{17}$ Selon une expression que nous empruntons à Georges Didi-Huberman, «Préface » à B. Prévost, La peinture en actes, op. cit., p. 14.

18 Selon l'expression de Léon Battista Alberti, dans le traité De Pictura.
} 
perception du mouvement qui n'est pas l'épreuve d'une succession de positions, mais "saisie englobante d'un accord sur notre harpe spatiale » (MSME, p. 187), autrement dit, la saisie d'un seul et même étant en mouvement. Si nous n'avions pas été dotés de cette faculté $\mathrm{d}^{\prime}$ anticiper le déroulement d'un mouvement sur une simple indication, les artistes n'auraient jamais pu, en composant des images immobiles, nous donner l'impression de la rapidité d'un déplacement. C'est pourquoi «l'impression du mouvement, et en conséquence de la vie, s'obtient beaucoup plus facilement à l'aide de quelques traits suggestifs que par le soin apporté dans la précision des détails $»^{19}$.

«Finalement, on parle de mouvement en peinture chaque fois que le monde est présenté indirectement, [...] à travers certains aspects obliques ou partiels ${ }^{20}$, par des formes ouvertes (typiques des tableaux du XVIIème siècle) qui "s'épaulent, s'entrelacent, se confondent " (MSME, p. 167). C'est précisément la solution que trouva Léonard de Vinci et que les Italiens appellent le sfumato : un contour enveloppant, des couleurs adoucies qui permettent aux formes de se perdre les unes dans les autres et nous communiquent l'imaginaire pulsation de la vie ${ }^{21}$.

L'on pourrait dire pour conclure, que l'impression du mouvement en peinture résulte d'un dessin vibrant et comme en suspens, "d'un éclat incertain" qui laisse son sillage dans le champ de la vision, balisant l'emplacement que le corps de l'objet avait occupé l'instant avant ${ }^{22}$, de sorte que tout mouvement résulterait de la prolongation ou de l'accompagnement d'un autre mouvement.

Peut-être il n'y a d'autre définition du mouvement que celle-là : quelque chose comme "l'insertion d'un mouvement dans un autre mouvement qui le précède » et « qui a déjà commencé dans son dos ${ }^{23}$. L'on peut citer à titre d'exemple le Discobole du sculpteur athénien Myron où le jeune athlète - représenté sur le point de lancer le lourd disque - se penche en arrière pour prendre son élan, avant de pivoter sur lui-même pour lancer le disque d'une détente de tout son corps.

\footnotetext{
${ }_{19}$ E. H. Gombrich, L'Art et l'illusion, op. cit., p. 191.

${ }^{20}$ M. Merleau-Ponty, Résumés de cours, Collège de France 1952-1960. Paris, Gallimard, 1964, p. 20, dorénavant noté $R C$.

${ }^{21}$ Cf. E. H. Gombrich, Histoire de l'art, Paris, Phaidon, 2006, p. 228.

22 Voir à cet égard le tableau Les Fileuses de Vélasquez qui paraît saisir ce que l'on nomme "l'effet stroboscopique ", le sillage indistinct que laisse dans le champ de vision le passage d'un objet en mouvement. Consulter l'analyse qu'y consacre E. H. Gombrich, L'Art et l'illusion, op. cit., p. 191.

${ }^{23} \mathrm{Cf}$. la référence indiquée note 16 .
} 
3. La formule charnelle du mouvement: le mouvement comme dunamis qui réunit en soi passivité et spontanéité, puissance et acte

Le problème se précise puisque l'on voit que la question devient : qu'est-ce que ce «mouvement qui a déjà commencé, ce mouvement qui se fait dans le dos $»^{24}$ ?

Une première réponse, assez simple pour l'instant, consiste à faire de ce mouvement qui nous précède, un donné naturel, ou plutôt le passé encore effectif et opérant de la perception, autrement dit le mouvement même de l'apparaître, de cette chair, celle $d u$ monde, qui nous précède et nous affecte du dedans. Simplement, ce passé, cette chair doit être ressaisie de façon dynamique, comme domaine des impressions qui nous met en mouvement en nous faisant répondre aux sollicitations qui nous sont livrées de manière sensible ; comme fond d'affectibilité grâce auquel le corps se ressent lui-même, et se constitue ainsi en un corpssujet opérant et actuel qui participe à l'émergence des possibilités inscrites dans le champ de l'apparaître par l'acte électif, de découpage et de sélection qu'il opère sur les perceptions qui lui sont ainsi données.

Plus profondément et radicalement encore, l'on devrait dire alors qu'au commencement de tous les mouvements il y a un mouvement qui engage et manifeste sans cesse la présence du corps, un mouvement charnel, qui advient de l'intérieur du monde et nous met en mouvement en nous faisant répondre à un appel qui nous incite à réaliser certaines possibilités, parmi celles qui nous sont livrées de manière sensible, par une action efficace sans laquelle les choses ne pourraient se montrer; un mouvement qui appartient au monde au moins autant qu'au sujet et qui est tout à la fois actif et passif, agissant et vécu comme résultat d'un contact affectif-impressionnel avec le monde qui éveille en nous une impulsion motrice ; un mouvement tout ensemble actif et passif, volontaire et subi, vécu et agi, qui prolonge l'activité $d u$ regard et y reconduit 25 ; un mouvement donc ressenti quoiqu'encore non réalisé, au cours duquel le sujet s'éprouve en même temps comme actif et passif.

\footnotetext{
${ }^{24}$ Cf. la référence indiquée note 16 .

${ }^{25}$ Ainsi, comme ces quelques lignes de Paul Klee - que Merleau-Ponty incorpore dans la trame de L'œil et l'esprit - le posent clairement : «Certain feu prétend vivre, il s'éveille; se guidant le long de la main conductrice, il atteint le support et l'envahit, puis ferme, étincelle bondissante, le cercle qu'il devait tracer : retour à l'oil et au-delà », OE, p. 58. Cette phrase dans sa formulation originale se retrouve peu différente in P. Klee, Théorie de l'art moderne, Paris, Gallimard, 1998, p. 36.
} 
En tant que tel, tout mouvement - venant de ce contact affectifimpressionnel avec le monde - ne signifie nullement quelque chose de subjectif, mais exprime au contraire la correspondance vécue entre le dedans et le dehors, entre les rythmes de la nature (les rythmes biologiques) et les rythmes qui habitent et meuvent notre propre corps, la conjonction rythmique $d^{\prime} u n$ se mouvoir et d'un être mû.

C'est cette ambiguïté fondamentale qui fait précisément tout l'intérêt de la définition du mouvement comme expression que nous propose Merleau-Ponty: le mouvement comme révélation, manifestation audehors d'un sens (encore captif, encore à-venir, présent seulement comme une esquisse naturelle) qui parle à notre corps en sollicitant de lui une résonance et un écho, le mouvement "comme un certain type de symbiose», une manière particulière "qu'a le dehors de nous envahir » à quoi correspond " une certaine manière que nous avons de $\mathrm{l}^{\prime}$ accueillir... $\gg^{26}$; en $\mathrm{d}^{\prime}$ autres termes, le mouvement comme invitation (sollicitation) adressée à notre corps de le prolonger à son gré, rayonnement d'une présence intensive, qui nous regarde et nous concerne, et tente nos capacités d'Einfühlung en tant qu'elle "fait vibrer notre regard, virtuellement notre toucher et nos oreilles ${ }^{27}$. Cette double intuition du mouvement, alimentée par l'expérience sensible (charnelle) donne lieu à un foisonnement d'images, de verbes et de métaphores dans ses textes ${ }^{28}$ qui développent les figures opposées de la passivité et de la tension active. On voit alterner le mouvement-rayonnement, vibration (expression), où sont les choses qui nous meuvent (parlent) ${ }^{29}$ et le mouvement-geste où l'être assume la forme volontaire d'un acte $\mathrm{du}$ corps, s'y retient et coïncide avec son expérience motrice dont l'effort (tension) se trouve récompensé par la perception presque érotique d'une énergie rassemblée où il pourra reconnaître l'autre bout de sa «puissance voyante » $(O E$, p. 16).

La combinaison de ces deux mouvements, leur conjonction rythmique, accomplie au plus haut niveau dans l'expression (acte d'expression) graphique ou parlante donne lieu à une véritable débauche d'événe-

\footnotetext{
${ }^{26}$ M. Merleau-Ponty, Phénoménologie de la perception (1945), Paris, Gallimard, 2003, p. 367. ${ }_{27}$ PM, p. 192.

${ }^{28}$ Cf. en particulier le chapitre de la Phénoménologie de la perception intitulé « Le Sentir » et plus précisément les passages consacrés à la perception des couleurs. Je renvoie tout particulièrement aux pages 245 et 247-48. Cf. également le chapitre de la Prose $d u$ monde intitulé "La perception d'autrui et le dialogue », le chapitre du Visible et l'invisible intitulé "L'entrelacs Le chiasme » et, enfin le chapitre II de L'œil et l'esprit, consacré à l'expérience de la vision picturale ou artistique.

29 «Toute chair, dit Merleau-Ponty, et même celle du monde, rayonne hors d'elle-même », $O E$, p. 55.
} 
ments esthétiques et de mouvements expressifs qui transfigurent la dynamique même de la chair du monde, produisant ainsi l'avènement de la culture (histoire) dans le même pli où c'est la nature qui s'exprime.

4. Du devenir-expressif de la chair du monde au «mouvement qui trace et laisse derrière lui les figures du sillage »: l'avènement de l'histoire

Finalement, tout cela suppose pour l'essentiel que l'histoire de l'art (et de la culture en général) se compose virtuellement par montage (combinaisons) de gestes comme un corps éloquent propre à gérer le récit d'un ensemble d'événements esthétiques, de devenirs expressifs qui transfigurent la dynamique même du visible (de la chair du monde, de l'apparaitre), comme un «corpus » d'images douées de mouvement qui, par déplacements successifs, s'étend d'un tableau à l'autre et qui noue enfin phénoménologiquement toutes ces images aux corps des spectateurs qui les regardent. D'où il en résulte :

...autant de convergences singulières qui font paraître, hors de toute influence, d'un bout à l'autre du monde des cuvres qui se ressemblent. ${ }^{30}$

Mais comment comprendre que cela soit possible?

Comment comprendre que des gestes représentés puissent nous émouvoir et même mouvoir le présent de nos propres gestes? Comment comprendre que des mouvements perçus (des formes en mouvement) puissent réveiller en nous les expériences qui les enracineront dans notre esprit ? Comment comprendre que des motions, des émotions figées comme par enchantement dans des gestes puissent traverser le temps et agiter, même mouvoir, le présent de nos propres gestes? Comment comprendre qu'une image puisse habiter, indivise, dans plusieurs esprits et dans plusieurs mondes?

Pour le comprendre, il faut d'abord remarquer que dans le procès de faire œuvre, le geste fait bien autre chose qu'arriver simplement, "venir" sous notre regard. Il présente notamment cette première particularité de se dépasser comme geste (comme mouvement) et «de signifier au-delà de sa simple existence de fait » (S, p. 110), de marquer une trace de son contact avec le monde, de déposer un témoignage de la forme en devenir en laquelle se configure cette rencontre. C'est en

${ }^{30}$ S, p. 108-109. 
cela qu'il «vaut au-delà de sa simple présence », « annonce une suite ou des recommencements $\gg(S$, p. 110$)$.

En ceci précisément qu'il «incorpore dans un tracé actif le schème d'une rencontre avec le monde $»^{31}$, " où tout autre regard retrouvera les motifs qui soutiennent son inspection du monde » $(O E$, p. 16) et dote ainsi les événements qui l'ont occasionné « de dimensions durables, par rapport auxquelles toute une série d'autres expériences auront sens, formeront une suite pensable ou une histoire [...] non pas au titre de survivance et de résidu, mais comme appel à une suite, exigence d'un avenir $»^{32}$.

C'est en cela, dira-t-on encore, qu'il est " par avance allié ou complice de toutes les autres tentatives d'expression» $(S$, p. 111). En ceci précisément que la transcendance $d u$ monde toujours à venir et imprévisible en son fond, "toujours derrière ce que je vois, en horizon » $(S$, p.37) ne cesse d'alimenter, mais aussi d'excéder sa manifestation visible à même la genèse de l'œuvre. Ainsi, tout geste, " en prise sur l'œuvre à faire », demeure "à la poursuite de ce qui, par delà sa dernière esquisse, le remet sans cesse en question, en demeure et en péril $»^{33}$, pour autant qu'il est « en résonance avec le regard, lui-même en prise sur la sur-prise du monde $»^{34}$.

De ce fait, l'histoire des formes (de la vision) qui en résulte ne reste jamais immobile, mais en appelle toujours à une suite, à un avenir et déclenche à son tour un travail de déplacement sans répit qui s'étend d'une image à l'autre, d'une forme visuelle à l'autre, d'un style à l'autre, d'une œuvre à l'autre. De même que les gestes qui l'ont produite ne possèdent pas la signification qu'ils vont créer sous la forme d'une fin donnée ou représentée à l'avance, de même l'histoire qui en résulte ne réalise pas un plan tout fait (elle "n'est pas gouvernée par un Esprit du Monde qui opérerait en nous sans nous », $S$, p. 106), mais elle n'est pas non plus une suite de hasards indépendants. Elle est l'ouverture d'un sens qui n'est pas donné d'avance et qui reste à faire: une évolution dont le sens reste indéterminé, et qui pourtant dans chaque création apporte avec elle la signification rétrospective de son propre passé - un passé sans cesse à redécouvrir qui est celui de la perception - de sorte que cette histoire se justifie rétrospectivement à partir d'un seul principe: le regard comme résonance et comme intuition, anticipation, pressentiment ou précession de ce qui est

\footnotetext{
${ }^{31}$ H. Maldiney, Art et existence, Paris, Klincksieck, 2003, p. 90.

${ }^{32}$ M. Merleau-Ponty, "L'institution dans l'histoire personnelle et publique », in L'institution, la passivité. Notes de cours au Collège de France (1954-1955), Paris, Belin, 1993, p. 124.

${ }^{33}$ H. Maldiney, Art et existence, op. cit., p. 90.

${ }_{34}$ Idem.
} 
sur ce qu'on voit, (OE, p. 58); un regard tourné vers la saisie vertigineuse des dimensions du réel qui dépassent le donné immédiat et capable ainsi d'embrasser l'horizon inépuisable auquel il appartient, de saisir le réel en totalité. Un regard animé par la volonté de voir non seulement tout ce qui est, mais aussi, sinon surtout, tout ce qui devient, de se rassembler dans une visée qui embrasse l'en-même-temps d'un seul coup.

Ici, comme partout, la seule règle est la vision. Simplement cette vision (la vision picturale ou artistique, en l'occurrence) n'est pas seulement un miroir toujours pareil à lui-même, mais une force de préhension vivante qui a sa propre "histoire interne ${ }^{35}$, ses formes de vision et de présentation propres, ses styles, ses schémas déterminés, et dont l'évolution compte plus d'une étape.

En ce sens, il faut dire à la fois que "le champ des significations picturales est ouvert depuis qu'un homme a paru dans le monde », que «le premier dessin aux murs des cavernes ne fondait une tradition que parce qu'il en recueillait une autre: celle de la perception » (S, p. 112-113) et que " chaque création change, altère, éclaire, approfondit, confirme, exalte, recrée ou crée d'avance toutes les autres ». (OE, p. 62). De chaque nouvelle création résulte en effet un mouvement qui se tourne et revient vers nous, celui de la reprise et de la re-création, selon les termes de Paul Klee. Dans ce mouvement de reprise, qui est celui de l'histoire proprement dite ( $S$, p. 112), chaque retour est un nouveau départ et amène avec soi son lot de changements, de variations, de surprises, des émotions de sorte que nous entendons le motif initial progresser dans la forêt des variations envisageables. C'est là précisément que résident la force et la grâce du mouvement qui trace, la nouveauté qu'il apporte : c'est le retour en possibilité de ce qui a été. La reprise restitue la possibilité de ce qui a été, le rend à nouveau possible, l'intensifie et le transforme en même temps en quelque chose $\mathrm{d}^{\prime}$ inattendu ${ }^{36}$. C'est là que réside précisément la proximité entre le mouvement qui trace et la mémoire. La mémoire restitue au passé sa possibilité, la possibilité d'être présent. Ce qui a été (le passé) cesse d'être un fait accompli (révolu) et redevient pour ainsi dire possible,

\footnotetext{
${ }^{35} \mathrm{Cf}$. à ce sujet $\mathrm{H}$. Wölfflin, Principes fondamentaux de l'histoire de l'art, Gérard Monfort, Brionne, 1984, et en particulier le paragraphe de la conclusion, intitulé "L'histoire interne et l'histoire externe de l'art », p. 257-259.

36 «Toute institution, dit Merleau-Ponty, comporte ce double aspect, fin et commencement, Endstiftung en même temps qu'Urstiftung. La sédimentation est cela : trace de l'oublié et par-là même appel à une pensée qui table sur lui et va plus loin ", L'institution. La passivité. Notes de cours au Collège de France (1954-1955), op. cit., p. 99.
} 
comme à-venir ou devenir de connaissance. Tel est le sens de la notion $\mathrm{d}^{\prime}$ institution chez Merleau-Ponty, laquelle « reprend une intention qui la précède et $[\ldots]$ en crée une qui lui survit et ira plus loin ${ }^{37}$. Tel est aussi le sens de cette expérience que Bergson voyait dans la mémoiresouvenir, lorsqu'il disait que le souvenir porte la trace du passé ; c'est la survivance virtuelle du passé qui peut à tout moment redevenir présent. La mémoire - au même titre que le mouvement faisant cuvre de signification - est pour ainsi dire l'organe de récréation du réel, ce qui peut transformer le passé en présent, le possible en réel et le réel en possible. L'on comprend alors pourquoi le mouvement qui trace peut avoir une telle importance historique, parce que c'est une façon de projeter la puissance et la possibilité du passé dans l'avenir.

Ce processus doit être expliqué aussi positivement par le fait que chaque image, chaque forme, chaque figure qui en résulte, est une fenêtre à travers laquelle la visibilité (ou bien la chair) du monde pénètre en nous et se cristallise en des formes déterminées. À chaque reprise, « en chaque nouveau style de la vision (artistique), un nouveau contenu de l'univers se cristallise ${ }^{38}$; un nouvel aspect de l'univers se révèle dans chaque forme nouvelle d'expression. On voit surgir d'un coup une foule de possibilités insoupçonnées qui bouleversent notre manière de voir, de sorte qu'il faudrait en conclure non seulement que l'on voit autrement, mais aussi que l'on voit autre chose. Et pourtant, chaque nouvelle forme est déjà contenue dans l'ancienne, de même que «le germe du feuillage nouveau se trouve déjà à côté du feuillage flétrissant ${ }^{39}$. Ce processus deviendra plus clair si l'on considère de près le passage qui conduisait de l'art égyptien aux « légendaires statues grecques qui rompent leurs entraves pour commencer à se mouvoir ${ }^{40}$, autrement dit le passage de la représentation linéaire qui caractérise l'art archaïque, où la forme en tant que telle s'impose fortement au spectateur, à la représentation picturale qui trouva les moyens de susciter l'impression du mouvement ${ }^{41}$.

\footnotetext{
${ }^{37}$ Ibid., p. 90.

${ }^{38}$ H. Wölfflin, Principes fondamentaux de l'histoire de l'art, op.cit., p. 276.

${ }^{39} \mathrm{Ibid} ., \mathrm{p} .269$.

${ }^{40}$ Selon une heureuse expression que nous empruntons à G. Agamben, « Notes sur le geste », in Moyens sans fins, Paris, Payot Rivages, 1995, p. 66.

${ }^{41}$ Une telle affirmation demande à être justifiée. Nous faisons ici allusion au changement de forme de la vision, à ce tournant décisif qui débouche sur l'art du XVIIe siècle et qui marque le passage de la représentation (style) linéaire à la représentation (style) picturale du mouvement, de la forme stable, immuable, dessinée avec un maximum de précision et avec une clarté absolue, qui caractérise l'art archaïque, le «hiératisme » de l'art primitif, où la forme en tant que telle s'impose fortement au spectateur, à la vision picturale qui trouva les moyens de susciter l'impression du mouvement, à travers notamment la technique du sfumato, du non-fini introduite par Léonard de Vinci. Cf. à ce sujet notamment $\mathrm{H}$. Wölfflin, Principes fondamentaux de l'histoire de l'art, op. cit.
} 
Ainsi, si nous regardons avec plus d'attention le Discobole,

\begin{abstract}
...nous découvrons que Myron a obtenu son extraordinaire effet de mouvement par un emploi nouveau de très anciennes méthodes. Si, face à cette statue, nous ne prenons garde qu'à ses contours, nous sommes frappés par son attachement à la tradition égyptienne. Comme les Égyptiens, Myron a présenté le torse de face, tandis que les jambes et les bras sont vus latéralement; comme eux, il a composé son auvre en combinant les aspects les plus caractéristiques des différentes parties du corps. Mais entre les mains de l'artiste, ces formules usées sont devenues quelque chose de tout différent. Au lieu d'assembler ces vues partielles en une pose rigide et peu naturelle, il a demandé à un modèle vivant de prendre à peu près cette attitude et il l'a modifiée de telle sorte qu'elle pût donner une image convaincante d'un corps en mouvement ${ }^{42}$.
\end{abstract}

Nous nous heurtons ici à un véritable paradoxe : celui d'une part, d'une forme qui s'avance au-delà de la précédente et anticipe ce qu'elle va devenir ; et, d'autre part, celui d'un motif qui revient, faisant entrer l'histoire dans le cercle de l'éternel retour, éternellement recommencé, par décentration et re-centration: d'un mouvement de retour et de recommencement qui n'est pas pour autant un cercle vicieux, mais au contraire une dunamis, une puissance infinie de re-création qui réalise le retour en possibilité de ce qui a été43.

Reste à déterminer plus précisément ce qu'est cette histoire circulaire où la fin est dans le commencement autant que le commencement est dans la fin. Une telle difficulté dépasse largement le cadre de notre enquête, et nous nous contenterons d'indiquer en passant la manière dont on pourrait selon nous la résoudre.

Cette histoire énigmatique semble bien relever de ce que MerleauPonty appelle l'« histoire des profondeurs », dont Bergson a eu, selon lui, l'intuition et que Péguy a développée : une histoire où «le sens se refait même au risque de se défaire », parce qu'il est "un sens volubile, bien conforme à la définition bergsonienne du sens qui est moins une chose pensée qu'un mouvement de pensée, moins un mouvement qu'une

\footnotetext{
${ }^{42}$ E. H. Gombrich, Histoire de l'art, op. cit., p. 77.

${ }^{43} \mathrm{Cf}$. à cet égard, le passage suivant : "...chez l'homme le passé peut non seulement orienter l'avenir ou fournir les termes des problèmes de l'adulte, mais encore donner lieu à une recherche au sens de Kafka, ou à une élaboration indéfinies : conservation et dépassement sont plus profonds, de sorte qu'il devient impossible d'expliquer la conduite par son passé, comme d'ailleurs par son avenir, qui se font écho l'un l'autre. L'analyse de l'amour chez Proust montre cette "simultanéité", cette cristallisation l'un sur l'autre du passé et de l'avenir [...]. L'institution d'une œuvre chez le peintre, d'un style dans l'histoire de la peinture, offre la même logique souterraine. Le peintre apprend à peindre autrement en imitant ses devanciers. Chacune de ses œuvres annonce les suivantes - et fait qu'elles ne peuvent pas être semblables ", M. Merleau-Ponty, L'institution. La passivité. Notes de cours au Collège de France (1954-1955), op. cit, p. 123-124.
} 
direction $(S$, p. 305). Une histoire «des profondeurs» dont l'essence réside dans la "jonction des individus et du temps », ou plutôt « dans la réponse d'une génération à ce qui fut commencé par une autre », dans " ce réseau d'appels et de réponses, où le commencement se métamorphose et s'accomplit » $(S$, p. 305) à la fois. Une histoire se faisant à travers une durée qui n'appartient en propre à personne, puisqu'elle coïncide avec "le rythme » même et «la vitesse propre de l'événement du monde » (S, p. 305).

Nous nous réservons de traiter plus amplement ce thème dans une autre étude : son examen représenterait ici un trop long détour. Mais qu'il nous suffise de dire, pour conclure, qu'il s'agit d'une histoire fondée sur une expérience intérieure et sur une compréhension en profondeur (de l'intérieur) du passé, de ce passé toujours présent et opérant de la perception qui diffuse et qui se communique à travers nous. Il s'agit en ce sens d'une connaissance intérieure, "sourde et profonde » de l'Être, de cet être oublié « qui contient par avance les vues, mêmes discordantes, même incompossibles, que nous pouvons en prendre, [...] et qui, une fois né, ne pourra jamais cesser d'avoir été, et qui continuera d'être au fond des autres présents » $(S$, p. 310$)$; une recréation vivante de ce passé, source de sens et de changement, création perpétuelle des formes mouvantes, grâce à laquelle sa poussée génératrice coule et irrigue le présent, substituant dans le travail de la mémoire le «se faisant» au «tout fait» historique. Une « histoire sans fin »- dira-t-on encore -, «destinée à forcer » par sa capacité inventive "les mystérieuses serrures des sens, dont la nature seule détenait primordialement la clef $»^{44}$.

\section{Conclusion}

C'est sur cette dernière remarque que nous souhaitons suspendre notre analyse pour en ressaisir le sens général. Elle nous ramène en effet au cœur de notre hypothèse de départ, hypothèse suivant laquelle tout mouvement, si spontané et créatif qu'il soit, doit s'insérer dans un autre mouvement plus fondamental qui «le précède » et «qui a déjà commencé dans son dos $»^{45}$ : le mouvement même de l'apparaître, de cette chair, celle $d u$ monde, qui nous précède et nous affecte $\mathrm{du}$ dedans. Un mouvement en lequel c'est la mobilité, l'imaginaire pulsation de la vie qui s'exprime et nous sollicite à réaliser certaines

\footnotetext{
${ }^{44}$ E. H. Gombrich, « De la représentation à l'expression » in L'Art et l'illusion, op. cit., p. 304.

${ }^{45} \mathrm{Cf}$. la référence indiquée note 16.
} 
possibilités, parmi celles qui nous sont livrées de manière sensible, par une action efficace sans laquelle elles ne pourraient pas même se manifester.

Se dévoile alors, à l'origine de tout mouvement, une résonance, voire une sonorité, plus encore une vibration qui saisit tous les lieux somatiques où résonne l'impulsion expressive d'un corps vivant qui vibre et se meut à l'écoute de tout ce qui est senti ou vécu au contact des "impulsions rythmiques", en provenance du donné - "celle d'une arête de montagne, d'un tronc d'arbre, des remous de l'eau, des ondes lumineuses $d^{\prime} u n$ visage ${ }^{46}$, des nuages dans le vent, des vagues dans la mer...

Ainsi, ce qui résonne au dos du mouvement, c'est une instance expressive, un désir d'exprimer et de s'exprimer, un vouloir-dire auquel il ne faut pas donner d'abord la valeur d'une volonté mais la signification inchoative d'une levée articulatoire - qu'elle soit profératrice ou graphique - encore sans intention et sans visée de signification, effleurant du fond de la cavité d'un corps vibrant, où s'amplifie la polyphonie même de la vie et se résout le mystère, voire le rythme profond d'une émotion intérieure, éprouvée au contact des mouvements de la nature.

Ce que Merleau-Ponty appelait le mouvement de l'expression et, dans les notes posthumes du Visible et l'invisible, la double incorporation du sensible et du sentant, ou bien leur "insertion réciproque », leur "entrelacs $» 47$, est probablement la logique la plus adéquate à expliquer la dynamique de cette résonance qui constitue le fond inouii, puisque toujours surprenant et inattendu, de tout mouvement qui trace que ce soit le trait du dessinateur, le signe de l'écrivain, l'articulation du discours (ce que Merleau-Ponty appelait dans la Préface à Signes citée en exergue, "le mouvement de la Parole ») et même la trame silencieuse de la pensée qui assure la continuité du flux énonciatif jusque dans les intervalles entre les signes (ce que Merleau-Ponty appelait « le mouvement de la Pensée ${ }^{48}$ ) - produisant l'avènement de l'histoire (de la culture) dans le même pli que celui où la nature s'exprime.

Dès lors, pour tirer toutes les conséquences de notre analyse, il faudra penser le mouvement selon le registre sonore comme élaboration et intensification de la disposition la plus tendue de l'écoute, ellemême auscultée au plus vif et au plus serré de sa tension et de sa

\footnotetext{
${ }^{46}$ Henri Maldiney, Art et Existence, op. cit, p. 90.

${ }^{47}$ M. Merleau-Ponty, Le visible et l'invisible, Paris, Gallimard, 1964, p. 182.

48 S, p. 36.
} 
pénétration, comme cette disposition profonde - disposée à l'affect selon la profondeur d'une caisse de résonance qui n'est autre que la cavité d'un corps vibrant de part en part et déjà en rapport à un sens qui lui échappe mais touche d'emblée son vécu; un corps tendu et presque aérien écoutant de tout son être un appel lui venant du monde et capable autant d'en retenir la résonance (l'écho qu'il éveille en lui), que d'en vocaliser, voire métamorphoser la trace. 